\title{
Anorexia nervosa e exercícios: questões éticas envolvendo profissionais de educação física
}

\section{Resumo}

A anorexia nervosa acomete cerca de $0,3 \%$ a $1 \%$ de mulheres jovens. A prática de exercícios em excesso é um comportamento problemático que ocorre em $40 \%$ a $80 \%$ dos indivíduos diagnosticados com esse transtorno alimentar. Para que suspeitem da doença os profissionais de educação física devem conhecer os vários comportamentos que essas pessoas adotam quando praticam atividade física, estar atentos tanto quanto aos riscos/benefícios da prática como às questões éticas decorrentes de aceitar treinar indivíduos nesta situação, pois, apesar de existirem poucos estudos sobre essas questões, há argumentos contrários e favoráveis quanto à indicação de exercícios. Em todo caso, o princípio básico deve ser o mesmo que se aplica a qualquer indivíduo que esteja praticando exercícios sob supervisão: promover a saúde.

Palavras-chave: Educação física e treinamento. Exercício. Esportes. Anorexia nervosa. Transtornos dismórficos corporais.

\section{Resumen}

\section{Anorexia nerviosa y ejercicios físicos: cuestiones éticas involucrando profesionales de educación física}

La anorexia nerviosa afecta alrededor del 0,3\% a $1 \%$ de mujeres jóvenes. La práctica de ejercicios en exceso es un comportamiento problemático que ocurre en $40 \%$ a $80 \%$ de los individuos diagnosticados con ese trastorno alimentario. Para que sospechen de la enfermedad los profesionales de educación física deben conocer los distintos comportamientos que esas personas adoptan cuando practican actividad física, estar atentos en cuanto a los riesgos/beneficios de la práctica así como a las cuestiones éticas derivadas por aceptar entrenar individuos en esta situación, puesto que, aunque existan pocos estudios a respecto de esas cuestiones, hay argumentos en contra y a favor de la indicación de ejercicios. De todos modos, el principio básico es el mismo que se aplica a cualquier individuo que practique ejercicios bajo supervisión: promover la salud.

Palabras-clave: Educación y entrenamiento físico. Ejercicio. Deportes. Anorexia nerviosa. Transtornos dismórficos corporales.

\section{Abstract}

\section{Anorexia nervosa and exercise: ethical issues involving physical education professionals}

Anorexia nervosa affects from $0.3 \%$ to $1 \%$ of young women. Excessive exercise is a problematic behavior that occurs in $40 \%$ to $80 \%$ of individuals with this eating disorder. Physical education professionals must observe multiple behaviors to make the suspicious of this disorder, be aware about the risks/benefits as well as the ethical issues of accepting to train people with anorexia nervosa because, even with few studies on this issue, there are favorable and contrary arguments regarding the indication of exercises. In any case, the basic principle is the same of what is applicable in any training practice under supervision: to promote health.

Key words: Physical education and training. Exercise. Sports. Anorexia nervosa. Body dysmorphic disorders.

Doutora luizacabus@hotmail.com - Universidade Federal da Bahia, Salvador/BA, Brasil.

Correspondência

Rua das Violetas, 66 apt $^{\circ} 602$ Pituba CEP 41810-080. Salvador/BA, Brasil.

Declara não haver conflito de interesse. 
A anorexia nervosa (AN) é uma doença psiquiátrica com alta morbimortalidade. Acomete principalmente mulheres jovens e caracteriza-se pelo medo intenso de ganhar peso, ainda que a pessoa esteja com a relação peso/altura inferior a $15 \%$ do esperado. Sua prevalência na população em geral varia de $0,3 \%$ a $1 \%{ }^{1}$.

Estima-se que $40 \%$ a $80 \%$ dos acometidos por essa patologia pratiquem exercícios com o objetivo de perder peso, o que mantém o círculo vicioso da doença, podendo levar a lesões físicas (inclusive permanentes) e, mesmo, à morte ${ }^{2}$. Ante a prevalência da $\mathrm{AN}$, da faixa etária que mais frequentemente ocorre (adolescentes e adultos jovens) e de sua associação com a prática excessiva de atividade física, é de se esperar que os profissionais de educação física (PEF) se deparem com indivíduos acometidos pela patologia. Portanto, devem estar capacitados para identificá-la.

Ainda que a patologia seja bem descrita na literatura, há pouca discussão sobre a supervisão assistida das atividades físicas nesse grupo específico de pacientes, porém temos que fazer menção aos estudos da autora Simona Giordano em suas reflexões sobre o tema ${ }^{3,4}$. Os médicos podem deixar de refletir sobre algumas questões importantes referentes à prática de exercícios assistidos; consideram-na, por vezes, parte integrante da doença e a proíbem. Nesse sentido, é fundamental que no tratamento da AN diversos profissionais estejam envolvidos, especialmente os PEF.

$O$ objetivo deste trabalho é refletir sobre questões éticas relacionadas à prática de exercícios supervisionados em pacientes com o diagnóstico - ou sob suspeita - de anorexia nervosa.

\section{Diagnóstico da AN e prática de exercícios em excesso}

A hiperatividade é um dos primeiros sinais da $A N$, surgindo antes mesmo que a perda de peso seja notada por familiares ou amigos. Considerando tal fato, o PEF pode ser um dos primeiros a notar, pela prática de exercícios com padrão peculiar, que não está lidando apenas com uma pessoa que quer meIhorar sua saúde ou performance. A atividade física praticada com o objetivo principal da perda de peso pode ser importante componente na patogênese e manutenção da AN ${ }^{5}$.

Essa característica - a hiperatividade - é fundamental para o diagnóstico precoce. É comum que pacientes com AN sejam diagnosticados apenas quando preenchem todos os critérios do DSM IV ou CID 10 (quadro anexo) ${ }^{6,7}$. Os indivíduos que não preenchem todos os critérios diagnósticos (a maioria) são classificados como portadores das chamadas formas parciais. Essas, no entanto, são tão graves quanto a forma clássica ${ }^{8}$.

Assim, os PEF devem estar atentos para alguns sinais e comportamentos que podem sinalizar a presença da AN:

- aparência emagrecida ou baixo peso à admissão (exame inicial) ou perda de peso e/ou massa muscular evidentes com o passar do tempo;

- ainda que perca peso, a pessoa diz que se alimenta bem. Observar que não ingere alimentos ou lanches antes ou depois da atividade física, mesmo que os colegas ou amigos o façam. Pode também recusar-se a ingerir água (há o falso pressuposto que beber água engorda);

- o aluno pode pesar-se repetidas vezes: antes, durante e depois da atividade física;

- observação minuciosa (por exemplo, em esteiras ou bicicletas) de quantas calorias está perdendo;

- o tempo no treino é longo e tende a aumentar a cada dia. Podem frequentar, se estiverem em uma academia, diversas aulas com diferentes professores para que não sejam notados. Preferem atividades aeróbicas e demonstram excessiva preocupação com a barriga, que pressupõem estar proeminente - o que, na maior parte dos casos, não corresponde à realidade;

- mudar o horário da atividade física se advertidos do excesso de horas de treino;

- usar várias roupas com o objetivo de suar mais e assim perder peso ou sentir mais frio que o esperado devido à escassez de gordura corporal;

- expressar repetidas vezes que estão gordos e que precisam resolver isso;

- interesse excessivo por exercícios que queimam mais calorias;

- idas frequentes ao banheiro para vomitar, urinar (abuso de diuréticos) e defecar (abuso de laxantes);

- câimbras repetidas, tontura e síncope;

- ansiedade excessiva por faltar um dia à academia ou treino. Necessidade de compensar a ausência;

- diante de lesões físicas repetidas e, mesmo com lesão decorrente do exercício, manter a prática. 
Embora detalhem os principais aspectos do comportamento compulsivo ligado à anorexia nervosa, os itens retrodescritos tornam evidente que o limite entre a normalidade e o comportamento abusivo é tênue em muitos casos. 0 padrão ideal socialmente difundido de perfeição associada à magreza, a ideia de que a prática de exercícios é sempre benéfica e o fato de que nem todo paciente com AN está em situação extrema de baixo peso são fatores que podem fazer com que a doença passe despercebida.

No entanto, não há consenso entre os pesquisadores sobre o que consiste a prática excessiva de exercícios. Não há consenso também em termos de número de horas ou dias por semana. O mais aceitável, conforme alguns autores parece relacionada à definição de prática em excesso, não é somente a quantidade de horas em si, mas a natureza obrigatória da atividade física e o sentimento de culpa se não cumprida ${ }^{9-11}$.

Na verdade, tal padrão revela-se muito semeIhante ao da dependência química. Nas duas situações ocorre a perda do controle que, no caso da AN, se manifesta por adotar exercícios mais intensos e/ ou por mais tempo em escala progressiva. Também para ambas fica evidenciado o comprometimento de atividades sociais e/ou acadêmicas. Além desses aspectos, indicam a possibilidade da prática excessiva de exercícios a manutenção da atividade ainda que haja lesão física persistente ou incapacidade devido à desnutrição ${ }^{12}$, situação que expressa falta de controle que acomete tanto o dependente químico quanto os pacientes com AN.

Para que o profissional de educação física identifique um provável portador de AN, é necessário observar não apenas a quantidade de horas que o indivíduo pratica atividade física sob sua supervisão. Deve estar atento, principalmente, à natureza compulsiva da pessoa, considerando a manutenção de esquema rígido e ritualístico de treinos e a manifestação de sensação de culpa quando não consegue cumprir integralmente o proposto.

\section{Riscos e benefícios da atividade física na AN e questões éticas}

Tanto aceitar quanto recusar a supervisão das atividades físicas de um indivíduo com AN são decisões que trazem implicações éticas. Essas se relacionam à beneficência e não maleficência e a questões morais e legais. Para que os PEF tomem a decisão de aceitar ou não paciente com AN em programa de exercícios físicos ou o treino em áreas específicas de competição, sugiro que algumas questões técnicas e éticas sejam levantadas, mesmo que nem todas as respostas sejam bem estabelecidas na literatura, a saber:

- quais as complicações da doença para o indivíduo?

- o exercício causa danos a quem tem AN?

- o exercício pode causar algum benefício nessa situação? Se o exercício é benéfico, qual o tipo, frequência e duração adequados?

- se o paciente apresentar alguma complicação leve ou grave durante a prática de exercícios, quais as consequências legais? Como deve o PEF se conduzir ante o risco iminente?

- se o paciente fizer exercícios sem supervisão, isso não aumentará seu risco de apresentar complicações?

- não seria eticamente mais correto fazer a supervisão da prática física do que deixar o paciente à mercê de sua patologia?

Para responder, é preciso saber que a AN está associada a muitas complicações. As mais significativas, no tocante ao maior risco durante a prática de exercícios, são bradicardia, hipotensão, arritmias, anormalidades hidroeletrolíticas (mais comuns em pacientes que purgam) e osteoporose ${ }^{13-15}$. As formas mais graves da doença apresentam alterações na frequência cardíaca, temperatura, pressão arterial e peso, que apontam a necessidade de internamento do paciente (tabela anexa) e - obviamente - contraindicam a prática de exercícios.

Entretanto, não há estudos suficientes no Brasil que avaliem a prática de exercícios como intervenção terapêutica em pacientes com AN, especialmente nos casos em que a doença se manifesta de forma mais branda. Há evidências de que a atividade física recomendada para adultos sadios nos Estados Unidos (EUA) - 150 minutos/semana de exercícios e musculação duas vezes/semana - não seja adequada para indivíduos com $\mathrm{AN}^{16}$. Mas não há qualquer recomendação acerca de qual tipo de exercício seja adequado para pacientes com AN; bem como não se sabe a intensidade em que devem ser praticados ou sua duração.

Daves et $a^{17}$, em estudo nacional realizado no Reino Unido, avaliaram por meio de entrevistas se as 66 unidades assistenciais que relatavam atender pacientes com AN possuíam consenso, protocolo ou guia para orientação de programa de atividades físicas supervisionadas para os pacientes que estavam 
internados. Quarenta e três (65\%) responderam o questionário elaborado pelos autores. Desses, 22 (51\%) referiram ter documentação escrita orientando a prática de exercícios e somente quatro $(9 \%)$ definiam por escrito o que seria a "prática saudável" de exercícios físicos.

Alguns critérios foram citados para que o indivíduo fosse liberado para a prática de exercícios em algumas das instituições, como, por exemplo, estar com IMC $>14 \mathrm{~kg} / \mathrm{m}^{2}$; ausência de complicações médicas significativas; manutenção do ganho de peso e praticar os exercícios apenas sob supervisão. Porém, o achado mais importante desse estudo foi que não havia consenso entre os diversos centros sobre os critérios adotados para a prática de exercícios e o tipo de intervenção que realizavam, indicando a falta de conhecimento específico sobre o assunto.

Calogero et al ${ }^{18}$ criaram programa de exercícios para pacientes internadas, com sessões de 60 minutos, quatro vezes por semana, incluindo exercícios de força, postura, yoga, uso de bolas, atividade aeróbica e jogos de recreação. As mulheres com AN que praticavam essa atividade física apresentaram ganho de peso $33 \%$ acima das que não o faziam. Apesar de incluir um número maior de pacientes com AN que outros estudos nessa área específica, o tempo de seguimento do estudo foi curto, o que dificulta consolidar os resultados.

Há, entretanto, algumas evidências de que as necessidades calóricas para ganho de peso em pacientes com AN que fazem exercício moderado não sejam diferentes daqueles que não o fazem. Contudo, os dados são insuficientes para indicar a prática de exercícios como parte do tratamento ${ }^{19}$. Apesar da ausência de recomendações nessa área específica, pacientes com AN que se exercitam em excesso dificilmente deixarão de praticar alguma atividade física quando melhoram clinicamente. Proibir o exercício tem se revelado medida inócua e impor esse tipo de restrição pode piorar a relação médico-paciente ${ }^{20}$.

Dado importante a ser considerado é que pacientes com transtorno alimentar (TA) tendem ao isolamento e a prática de exercícios pode facilitar as relações sociais, melhorar a autoestima, a imagem corporal e o humor ${ }^{21}$. Para Giordano ${ }^{3}$, há também razões éticas para permitir que pacientes com TA pratiquem atividades físicas supervisionadas: 1 ) preservar a autonomia do paciente respeitando suas escolhas individuais; 2) minimizar o dano a curto prazo: ou seja, proteger contra consequências mais sérias que poderiam advir da ausência de supervisão; 3) minimizar o dano a longo prazo: prevenir a sensação de perda de autonomia e controle; 4) promover $\mathrm{o}$ autocontrole e responsabilidade.

Existem razões éticas para permitir que pessoas com AN pratiquem exercício, mas não há obrigação moral do PEF exercer a função de supervisão ${ }^{22}$. Há vários motivos técnicos e éticos que podem ser alegados pelos profissionais para não aceitar esses indivíduos como participantes de atividades físicas. O mais significativo é o PEF referir que não possui formação para tal. Os treinos de esportes e as aulas em academias são desenhadas ou preparadas para pessoas que teoricamente não correm riscos evidentes com a prática. No caso dos portadores de AN, a prática de exercícios físicos pode contribuir para o agravamento da doença quer através da perda de peso ou de lesões decorrentes do esforço, situação que colocaria os PEF em posição eticamente delicada.

Há entre os PEF aqueles capacitados e treinados para trabalhar com situações específicas como idosos ou gestantes. No entanto, são situações diferentes, visto que a especificidade da condição não é escondida. Nesses casos, o PEF conhece os riscos potenciais e pode orientar as atividades apropriadas. Para esses grupos há inequívocos benefícios decorrentes do exercício físico planejado, o que ainda não se pode afirmar em relação a AN.

A recusa do profissional de educação física em trabalhar com indivíduos com AN pode se dar ainda que saiba o diagnóstico. Pode argumentar que dada à natureza compulsiva do exercício praticado por pessoas com essa patologia, riscos como o de maior perda de massa muscular e peso são ampliados. Esse processo pode levar à amenorreia e complicações como osteopenia e osteoporose, que, por sua vez, podem propiciar fraturas ${ }^{15}$. Pode não aceitar trabalhar com anorética(o)s argumentando que seu trabalho pode acarretar malefício ao indivíduo, ao invés do benefício esperado.

As duas principais causas de morte na anorexia são o suicídio e alterações cardíacas. O risco iminente de arritmia cardíaca fatal tem que ser levado em conta. A maior parte dos adolescentes e crianças admitidos em emergências com complicações da AN o fazem devido a anormalidades cardíacas ${ }^{23}$. Diante disso, o profissional pode recusar a supervisão porque precisa contar com suporte técnico (aparelho de cardioversão) e treinamento para lidar com caso de emergência.

O PEF está prestando um serviço como o médico, o advogado ou o psicólogo e pode não se sentir apto a realizá-lo. Esse é um direito e um dever 
inconteste do profissional. Moralmente, explicar os motivos da recusa se impõe. Direcionar esse indivíduo a procurar ajuda médica especializada é de extrema importância. Se menor de idade, é obrigação do PEF comunicar seus responsáveis legais. Nessa situação, o adolescente ou a criança podem ser avisados de que seus pais serão acionados a depender de cada caso. Documentar os fatos na ficha de atendimento é fundamental porque legalmente estará respaldado caso ocorra algum problema posterior. Se os responsáveis legais não comparecerem para ter conhecimento dos fatos e havendo suspeita de risco de vida iminente, cabe ao profissional acionar o conselho tutelar.

Ainda que haja atestado médico dizendo que o menor ou até um adulto com AN pode praticar a atividade física, o PEF deve rever essa liberação com o passar do tempo. Precisa estar atento para o fato de que em alguns dias ou semanas a condição da pessoa pode vir a deteriorar e o médico pode não ter sido avisado ou os pais não terem notado. Nesse caso, cabe ao profissional agir em prol da saúde do indivíduo, avisando-o ou a seus familiares dos prováveis danos relacionados à atividade física em tais condições.

Assim, diante de tantas incertezas e questionamentos, o que seria ideal? Como em qualquer área que lide com a saúde, os melhores resultados sempre são obtidos a partir do trabalho em conjunto: que o PEF tivesse acesso direto aos médicos, psicólogos e aos pais e que todos, juntos, avaliassem a situação para propor a melhor estratégia, obviamente consultando também a pessoa com AN. Dessa forma, é possível encontrar respostas e adotar comportamentos que promovam o bem-estar e a autonomia do indivíduo, restituindo a saúde e salvaguardando sua vida.

\section{Considerações finais}

Pelo exposto, é relevante que os PEF discutam sobre aceitar ou não que pessoas com AN pratiquem exercícios ou esportes sob supervisão. A questão torna-se ainda mais crucial porque indivíduos nessa situação tendem a usar a prática da atividade física como mecanismo de perda de peso e, assim, perpetuar um ciclo que agrava a doença.

É provável que centenas ou milhares de pessoas acometidas pela AN frequentem academias e treinos de esportes, sem que os devidos cuidados sejam tomados. Sendo assim, o profissional de educação física deve estar apto a identificar sinais e comportamentos que possam sugerir a presença de patologia grave como essa.

A falta de dados na literatura sobre o papel do exercício causando benefício no tratamento da patologia cria um dilema. Não existe resposta única e definitiva. Ante isso, é importante promover a discussão interdisciplinar para refletir sobre a complexidade da situação e encontrar a melhor alternativa para todos os envolvidos. Ao tomar a atitude de aceitar ou não que pessoas com AN pratiquem atividades físicas, o PEF precisa estar ciente de que assume riscos e consequências tanto aceitando quanto recusando a supervisão. Essa decisão, portanto, deve ser respaldada em conhecimento sobre o tema e em discussão interdisciplinar, objetivando a promoção da saúde e bem-estar dos portadores de anorexia nervosa.

\section{Referências}

1. Keski-Rahkonen A, Hoek HW, Linna MS, Raevuori A, Shivola E, Bilik CM et al. Epidemiology and course of anorexia nervosa in the community. Am J Psychiatry. 2007;164(8):1.259-65.

2. Zunker C, Mitchell JE, Wonderlich SA. Exercise interventions for women with anorexia nervosa: a review of the literature. Int J Eat Disord. 2011;44(7):579-84.

3. Giordano S. Exercise and eating disorders an ethical and legal analysis. New York: Routhledge; 2010.

4. Giordano S. Understandig eating disorders: conceptual and ethical issues in the treatment of anorexia and bulimia nervosa. New York: Oxford University Press; 2005.

5. Hechler T, Beumont P, Marks T, Touyz S. How do clinical specialists understand the role of physical activity in eating disorders? Eur Eat Disord Rev. 2005;13(2):125-32.

6. American Psychiatry Association. Diagnostic and statistical manual of mental disorders. $4^{\text {th }}$ ed. Washington: APA; 1994.

7. World Health Organization. The ICD-10 classification of mental and behavioural disorders: diagnostic criteria for research. Geneva: WHO; 1993.

8. Ackard DM, Fulkerson JA, Neumark-Sztainer D. Prevalence and utility of DSM-IV eating disorder criteria among youth. Int J Eat Disord. 2007;40(5):409-17.

9. Boyd C, Abraham S, Luscumbe G. Exercise behaviors and feelings in eating disorder and noneating disorder groups. Eur Eat Disord Rev. 2007;15(2):112-8. 
10. Cook BJ, Hausemblas HA. The role of exercise dependence for the relationship between exercise behavior and eating pathology: mediator or moderator? J Health Psychol. 2008;13(4):495-502.

11. Scroff H, Reba L, Thornton LM, Tozzi F, Klump KL, Barrettini WH et al. Features associated with excessive exercise in women with eating disorders. Int J Eat Disord. 2006;39(6):454-61.

12. Klein DA, Bennett A, Schebendach J, Foltin RW, Devlin MJ, Walsh T. Exercise "addiction" in anorexia nervosa: model, development and pilot data. CNS Spectrum. 2004;9(7):531-7.

13. Palla B, Litt IF. Medical complications of eating disorders in adolescents. Pediatrics. 1988;81(5):61323.

14. Swenne I, Larsson PT. Heart risk associated with weight loss in anorexia nervosa and eating disorders: risk factors for QTc interval prolongation and dispersion. Acta Pediatr. 1999;88(3):304-9.

15. Golden NH. Osteopenia and osteoporosis in anorexia nervosa. Adolesc Med. 2003;14(1):97-108.

16. US Department of Health and Human Services. Physical Activity Guidelines for Americans. [Internet]. Washington: HHS; 2008 (acesso 28 abr. 2012). Disponível: http://www.health.gov/ paguidelines/pdf/paguide.pdf

17. Daves S, Parech K, Etelepaa K, Wood D, Jaffa T. The impatient management of physical activity in young people with anorexia nervosa. Eur Eat Disord Rev. 2008;16(5):334-40.

18. Calogero R, Pedrotty K. The practice and process of health exercise: an investigation on the treatment of exercise abuse in women with eating disorders. Eat Disord. 2004;112(4):273-91.

19. Hausemblas HA, Cook BJ, Chittester NI. Can exercise treat eating disorders? Exerc Sport Sci Rev. 2008;36(1):43-7.

20. Beumont PJV, Arthur B, Russell JD, Touyz SW. Excessive physical activity in dieting disorder patients: proposal for a supervisioned exercise program. Int J Eat Disord. 1994;15(1):21-36.

21. Fossati M, Amati F, Painor D, Reiner M, Haemi C, Golay A. Cognitive-behavioral therapy with simultaneous nutritional and physical activity education in obese patients with binge eating disorder. Eat Weight Disord. 2004;9(2):134-8.

22. Giordano S. Risk and supervisioned exercise: the example of anorexia to illustrate a new ethical issue in the traditional debates of medical ethics. J Med Ethics. 2005;31:15-20.

23. Miller KK, Grinspoon SK, Ciampa J, Hier J, Herzog D, Kimbamki A. Medical findings in outpatients with anorexia nervosa. Arch Intern Med. 2005;165(5):561-6.

24. Sylvester CJ, Forman SF. Clinical practice guidelines for treating restrictive eating disorder patients during medical hospitalization. Curr Opin Pediatr. 2008;2008(4):390-9.

25. Golden NH, Katzman DK, Kreipi RE, Stevens SL, Sawyer SM, Rees J et al. Eating disorders in adolescents: position paper of The Society for Adolescent Medicine. J Adolesc Health. 2003;33(6):496- 503.

26. American Academy of Pediatrics. Committee on Adolescence Identifying and Treatment Eating Policy Statement. Pediatrics. 2003;111(1):204-11.

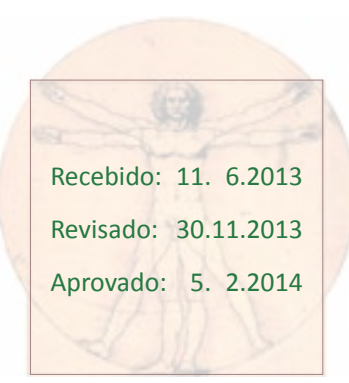




\section{Anexo}

Quadro. Critérios diagnósticos para anorexia nervosa segundo o DSM-IV e a CID-10

DSM-IV
A. Recusa a manter o peso corporal em um nível igual ou
acima do mínimo normal adequado à idade e à altura (por
ex., perda de peso levando à manutenção do peso corpo-
ral abaixo de $85 \%$ do esperado; ou fracasso em ter o ga-
nho de peso esperado durante o período de crescimento,
levando a um peso corporal menor que $85 \%$ do esperado).

B. Medo intenso de ganhar peso ou de se tornar gordo, mesmo estando com peso inferior.

C. Perturbação no modo de vivenciar o peso, tamanho ou formas corporais; excessiva influência do peso ou da forma corporais na maneira de se autoavaliar; negação da gravidade do baixo peso.

D. No que diz respeito especificamente às mulheres, a ausência de pelo menos três ciclos menstruais consecutivos, quando é esperado ocorrer o contrário (amenorreia primária ou secundária). Considera-se que uma mulher tem amenorreia se os seus períodos menstruais ocorrem somente após o uso de hormônios, por exemplo, estrógeno administrado.

Tipo:

- Restritivo: não há episódio de comer compulsivamente ou prática purgativa (vômito autoinduzido, uso de laxantes, diuréticos, enemas)

- Purgativo: existe episódio de comer compulsivamente e/ ou purgação.

\section{CID-10}

A. Há perda de peso ou, em crianças, falta de ganho de peso, e o peso corporal é mantido em pelo menos $15 \%$ abaixo do esperado.

B. A perda de peso é autoinduzida pela evitação de "alimentos que engordam".

C. Há distorção na imagem corporal na forma de uma psicopatologia específica de um pavor de engordar.

D. Um transtorno endócrino generalizado envolvendo o eixo hipotalêmico hipofisário gonodal é manifestado em mulheres como amenorreia e em homens como uma perda de interesse e potências sexuais (uma exceção aparente é a persistência de sangramentos vaginais em mulheres anoréxicas que estão recebendo terapia de reposição hormonal, mais comumente tomada como uma pílula anticonceptiva).

Comentários: se o início é pré-puberal, a sequência de eventos da puberdade é demorada ou mesmo detida (o crescimento cessa; nas garotas, as mamas não se desenvolvem e há uma amenorreia primária; nos garotos, os genitais permanecem juvenis). Com a recuperação, a puberdade é com frequência completada normalmente, porém a menarca é tardia; os seguintes aspectos corroboram o diagnóstico, mas não são elementos essenciais: vômitos autoinduzidos, purgação autoinduzida, exercícios excessivos e uso de anorexígenos e/ou diurético.

Tabela. Indicações de internamento em adolescentes com anorexia nervosa

\begin{tabular}{|c|c|c|c|}
\hline Critério & $\begin{array}{l}\text { Sociedade de Medicina da } \\
\text { Adolescência (EUA, 2003) }{ }^{24}\end{array}$ & $\begin{array}{l}\text { Academia Americana de } \\
\text { Pediatria (EUA, 2003) }\end{array}$ & $\begin{array}{l}\text { Associação Americana de } \\
\text { Psiquiatria (EUA, 2006) }{ }^{26}\end{array}$ \\
\hline Peso & $\begin{array}{c}\leq 75 \% \text { do ideal } \\
\text { para a altura }\end{array}$ & $\begin{array}{c}\leq 75 \% \text { do ideal } \\
\text { para a altura }\end{array}$ & $\begin{array}{l}\leq 85 \% \text { do peso sadio } \\
\text { Perda aguda com recusa } \\
\quad \text { em se alimentar }\end{array}$ \\
\hline $\begin{array}{l}\text { Frequência } \\
\text { cardíaca }\end{array}$ & $\begin{array}{l}<50 \mathrm{bpm} \text { de dia } \\
<45 \mathrm{bpm} \text { à noite }\end{array}$ & $\begin{array}{l}<50 \mathrm{bpm} \text { de dia } \\
<45 \mathrm{bpm} \text { à noite }\end{array}$ & $40 \mathrm{bpm}$ \\
\hline $\begin{array}{l}\mathrm{TA}(\mathrm{mmHg}) \\
\text { alterações } \\
\text { ortostáticas }\end{array}$ & $<80 / 50$ & sistólica $<90$ & $<80 / 50$ \\
\hline 个 FC & $>20$ & $>20$ & $>20$ \\
\hline$\downarrow$ TA & $>10$ & $>10$ & $>20$ \\
\hline Temperatura & $<36^{\circ} \mathrm{C}$ & $<35,6^{\circ} \mathrm{C}$ & $<36,1^{\circ} \mathrm{C}$ \\
\hline Eletrólitos & Hipocalemia & $\mathrm{K}<3.2 \mathrm{mEq} / \mathrm{L}$ & Hipofosfatemia \\
\hline & $\begin{array}{l}\text { Hipofosfatemia } \\
\text { Hiponatremia }\end{array}$ & $\mathrm{Cl}<88 \mathrm{mEq} / \mathrm{L}$ & Hipomagnesemia \\
\hline Gerais & $\begin{array}{c}\text { Falha no tratamento } \\
\text { ambulatorial }\end{array}$ & $\begin{array}{c}\text { Falha no tratamento } \\
\text { ambulatorial }\end{array}$ & $\begin{array}{c}\text { Falha no tratamento } \\
\text { ambulatorial }\end{array}$ \\
\hline
\end{tabular}

